Classification

Physics Abstracts

$61.12-61.30-61.40 \mathrm{~K}$

\title{
Influence of the strength of the smectic order on the backbone anisotropy of side-chain liquid crystal polymers as revealed by SANS
}

\author{
L. Noirez, P. Keller and J. P. Cotton \\ Laboratoire Léon Brillouin (CEA-CNRS), CE-Saclay, 91191 Gif-sur-Yvette Cedex, France
}

(Received 20 December 1991, accepted in final form 10 February 1992)

\begin{abstract}
Résumé. - Il est proposé que la qualité de l'ordre smectique détermine l'anisotropie du squelette de polymères mésomorphes en peigne confinés dans les lamelles. Ici l'ordre smectique est augmenté en allongeant le groupe alkyl terminal des mésogènes. Nous étudions deux polyméthacrylates cristal liquide qui ne different que par leurs groupes terminaux: $-\mathrm{OCH}_{3}$ et $-\mathrm{OC}_{4} \mathrm{H}_{9}$. L'anisotropie du squelette est mesurée par diffusion de neutrons aux petits angles tandis que l'ordre smectique est évalué à l'aide de l'intensité du pic de Bragg 001 .
\end{abstract}

\begin{abstract}
It is proposed that the strength of the smectic order determines the backbone anisotropy of side-chain liquid crystal polymers. Here this strength increases with the length of the alkyl terminal group of the mesogens. Two liquid crystal polymethacrylates differing only by the mesogenic tails $-\mathrm{OCH}_{3}$ and $-\mathrm{OC}_{4} \mathrm{H}_{9}$ are considered. The backbone anisotropy of these polymers is measured by small angle neutron scattering (SANS) whereas the smectic order is evaluated from the intensity of the 001 Bragg peak.
\end{abstract}

\section{Introduction.}

By grafting liquid crystal molecules on the side of a polymer chain via a spacer, one can obtain a side-chain liquid crystal polymer which presents the usual mesophases as the nematic or the smectic phase. The structure of the mesophase can be characterized by diffraction [1] of Xrays or neutrons and the conformation of the polymer by small angle scattering [2] of neutrons [3]. Since a mixture of all hydrogenated liquid-crystal polymers with liquid crystal polymers deuterated on the backbone produces [3] a signal at small angles by neutron scattering from which the polymer backbone conformation can be deduced.

Here we compare the backbone conformation of two liquid-crystal polymethacrylates of closed polymerization degrees and chemical formula. The only difference is the length of the alkyl tail of each mesogen which is $\mathrm{OCH}_{3}$ for the first polymer called $\mathrm{PMA}-\mathrm{OCH}_{3}$ and $\mathrm{OC}_{4} \mathrm{H}_{9}$ for the second one. To understand the difference of the anisotropy ratio, we analyze the diffraction pattern at small angles (001 Bragg peak) and propose to relate the confinement of the polymer backbone to the smectic order strength measured by the intensity of the 001 Bragg reflection. 


\section{Materials and experimental approach.}

2.1 ChARACTERISTICS OF THE LIQUID-CRYSTAL POLYMERS. - The two liquid-crystal polymers $\mathrm{PMA}-\mathrm{OCH}_{3}$ and $\mathrm{PMA}-\mathrm{OC}_{4} \mathrm{H}_{9}$ as well as their homologous deuterated on the backbone are synthetized [4] via radical polymerization of their liquid-crystal monomers.

The liquid-crystal polymethacrylate called $\mathrm{PMA}-\mathrm{OCH}_{3}$. - $\mathrm{PMA}-\mathrm{OCH}_{3}$ corresponds to the following formula

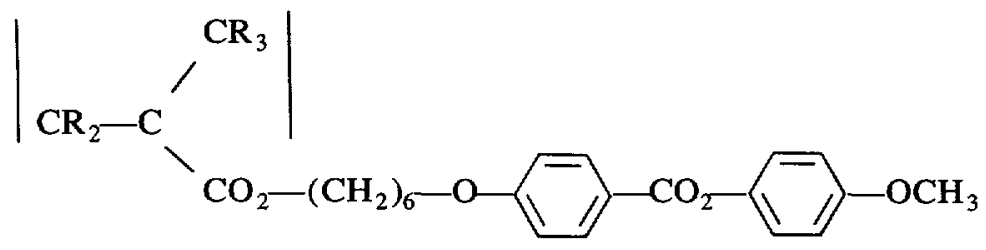

where $\mathrm{R} \equiv \mathrm{H}$ or $\mathrm{D}$.

The polymerization degree in weight is $\mathrm{DP}_{\omega} \simeq 800$ and its polydispersity $I=2.7$.

It presents the isotropic $(\mathrm{I})$-nematic $(\mathrm{N})$-smectic $\left(\mathrm{S}_{\mathrm{A}}\right)$-glassy $\left(\mathrm{T}_{\mathrm{g}}\right)$ succession of phases with the respective temperatures :

$$
\mathrm{I}-106^{\circ} \mathrm{C} \mathrm{N}-73^{\circ} \mathrm{C} \mathrm{S} \mathrm{S}_{\mathrm{A}}-40{ }^{\circ} \mathrm{C} \mathrm{T} \text {. }
$$

This smectic phase has not always been identified in the literature [5] nevertheless diffraction study [6] reveals a well-defined smectic phase of monolayer structure (the smectic layer thickness is $25 \pm 1 \AA$ and corresponds to the mesogenic length in its extended form).

The liquid-crystal polymethacrylate called $\mathrm{PMA}-\mathrm{OC}_{4} \mathrm{H}_{9}-\mathrm{PMA}-\mathrm{OC}{ }_{4} \mathrm{H}_{9}$ is a polymethacrylate identical to PMA-OCH ${ }_{3}$ excepted for the alkyl end of the mesogens which is substituted by $\mathrm{OC}_{4} \mathrm{H}_{9}$. It has then the following formula :

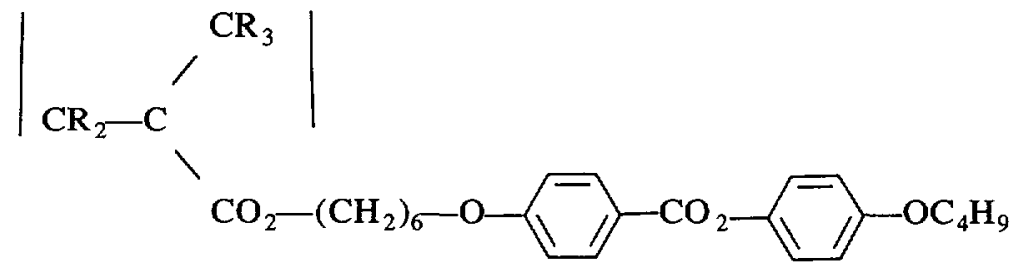

where $\mathrm{R}=\mathrm{H}$ or $\mathrm{D}$.

The degree of polymerization in weight is $\mathrm{DP}_{\omega}=680$ and is comparable to that of PMA$\mathrm{OCH}_{3}$. The phase succession is the same as for $\mathrm{PMA}-\mathrm{OCH}_{3}$, but the substitution of the terminal group $\mathrm{OCH}_{3}$ by $\mathrm{OC}_{4} \mathrm{H}_{9}$ induces a shrinking of the nematic temperature range :

$$
\mathrm{I}-115^{\circ} \mathrm{C} \mathrm{N}-108^{\circ} \mathrm{C} \mathrm{S}-42^{\circ} \mathrm{C} \mathrm{T} \text {. }
$$

The smectic phase is also a monolayer smectic phase $\left(S_{A 1}\right)$ since the layer thickness has the same value as the most elongated size of the mesogen (28-29 $\AA$ ).

\subsection{EXPERIMENTAL DETAILS.}

2.2.1 Sample environment. - The sample is a disk-like quartz cell of $15 \mathrm{~mm}$ diameter and $1 \mathrm{~mm}$ thickness filled with a mixture $1: 1$ of all hydrogenated liquid crystal polymers with liquid crystal polymers deuterated on the backbone. This sample is thermalized in an oven itself put between the poles of a magnet. The trajectory of the incident neutrons cross the faces of the sample and the scattered beam is collected on the plane of a 2-dimension 
multidetector (Small Angle Neutron Scattering Device PAXY of the Laboratoire Léon Brillouin in the Orphée Reactor). In this geometry, the intensity collected by the multidetector corresponds to a scattering plane containing the directions parallel and perpendicular to the magnetic field. Using the classical procedure $[7,8]$, we determine the two components of the radius of gyration of the polymer backbone $R_{\|}$and $R_{\perp}$ respectively parallel and perpendicular to the magnetic field direction.

2.2.2 The thermal procedure. - The sample is heated to the isotropic phase, and then slowly cooled from this isotropic state under an applied a magnetic field of $1.4 \mathrm{~T}$. The monodomains of the mesophase are realigned in the nematic phase at a temperature closed to the isotropic nematic transition. We check that the alignment is good by the optical transparency of the sample and the invariance of the values of $R_{\|}$and $R_{\perp}$ after two successive measurements at a same temperature. The experiment is carried on by the systematic determination of $R_{\|}$and $R_{\perp}$ for a series of temperatures obtained by cooling till the glassy state. Otherwise, we determine the nematic-smectic transition and the quality of the alignment by the appearance and the thickness of 001 Bragg peak.

2.2.3 Scattering laws: theory and application to the liquid-crystal polymers. - It has been demonstrated that the neutron scattered by a mixture in bulk of hydrogenated and deuterated polymers produces a small angle scattering associated to interferences between pairs of scatterers belonging to a same chain [9]. This intensity is proportional to the form factor $P(q), q$ is the module of the scattering vector, of the backbone which is deuterated part of the polymers. By calling $R_{\mathbb{I}}$ and $R_{\perp}$ the components of the radius of gyration of the backbone in the directions parallel $(l l)$ and perpendicular $(\perp)$ to the magnetic field, one has :

$$
\begin{aligned}
& P^{-1}\left(q_{\|}\right)=P^{-1}(0) \cdot\left(1+q_{\|}^{2} \cdot R_{\|}^{2}\right) \\
& P^{-1}\left(q_{\perp}\right)=P^{-1}(0) \cdot\left(1+q_{\perp}^{2} \cdot R_{\perp}^{2}\right)
\end{aligned}
$$

These relations are valid in the Guinier range $\left(q R_{\|}\right.$and $\left.q R_{\perp} \leqslant 1\right)$ and allows the determination of $R_{\|}$and $R_{\perp}$.

Two scattering domains have been used :

- the first one corresponding to $0.008 \AA^{-1}<|\mathbf{q}|<0.07 \AA^{-1}$ permits the access to the small-angle scattering domain. The wavelength used was $15 \AA$ and the distance samplemultidetector $2 \mathrm{~m}$,

- the second one was obtained just by switching the wavelength down to $3.7 \AA$ in order to observe the 001 Bragg reflection $0.04 \AA^{-1}<|\mathbf{q}|<0.13 \AA^{-1}$

\section{Results.}

The evolution of the backbone conformation of $\mathrm{PMA}-\mathrm{OCH}_{3}$ and $\mathrm{PMA}-\mathrm{OC}_{4} \mathrm{H}_{9}$ is drawn in the same figure, figure 1 , by using reduced parameters :

$$
r_{\|}=\frac{R_{\|}}{R_{\text {iso }}} \quad \text { and } \quad r_{\perp}=\frac{R_{\perp}}{R_{\text {iso }}}
$$

for the radii of gyration where $R_{\text {iso }}$ is the value determined in the isotropic phase ( $63 \AA$ for PMA$\mathrm{OC}_{4} \mathrm{H}_{9}$ and $69 \AA$ for $\left.\mathrm{PMA}-\mathrm{OCH}_{3}\right)$. The reduced temperature is $\left(T-T_{\mathrm{NS}}\right) / T_{\mathrm{NS}}$ where $T_{\mathrm{SN}}$ is the temperature of the nematic-smectic transition.

The two polymers present from the isotropic nematic transition an oblate shape for which the polymer backbone is in average perpendicular to the main direction of the liquid crystals $\left(R_{\perp}>R_{\|}\right)$. This oblate deformation is weak in the nematic phase and becomes stronger when passing in the smectic phase. 


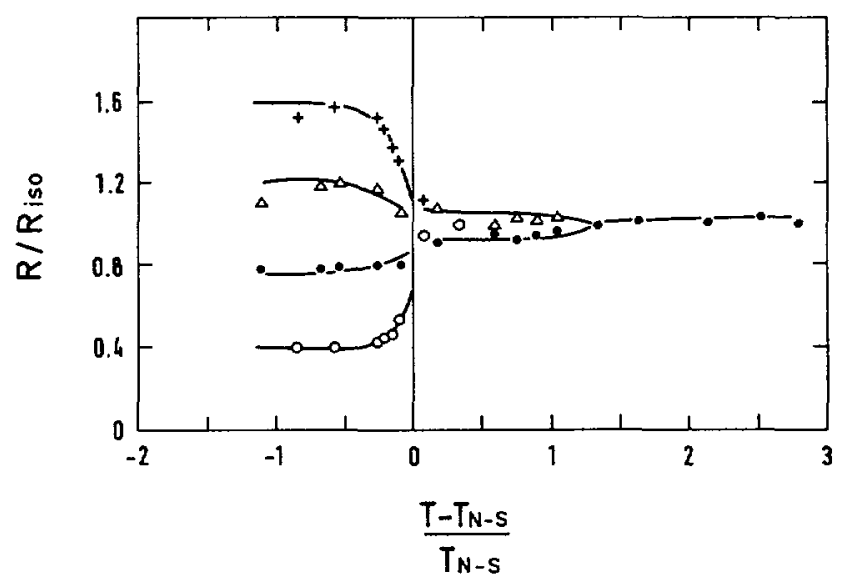

Fig. 1. - Plot of the reduced values $\left(r_{I}\right.$ and $r_{\perp}$ ) of the radius of gyration, parallel $(\|)$ and perpendicular $(\perp)$ to the magnetic field, versus the reduced temperature. The data of $\mathrm{PMA}^{-\mathrm{OCH}_{3}}$ ( $\bullet$ for $r_{1}, \Delta$ for $\left.r_{\perp}\right)$ can be compared to those of PMA-OC ${ }_{4} \mathrm{H}_{9}\left(O\right.$ for $r_{1}, \Delta$ for $\left.r_{\perp}\right)$.

It appears clearly from figure 1 that the backbone of $\mathrm{PMA}-\mathrm{OCH}_{3}$ is largely less confined in the smectic phase, than the backbone of $\mathrm{PMA}-\mathrm{OC}_{4} \mathrm{H}_{9}$. However, the deformation acts in the same way and conserves the direction of the anisotropy, only the deformation ratio changes.

\section{Interpretation.}

An interpretation based on the polymer characteristics like the polymerization degree, the rigidity of the backbone chain must be excluded since the only difference between PMA$\mathrm{OCH}_{3}$ and $\mathrm{PMA}-\mathrm{OC}_{4} \mathrm{H}_{9}$ reads in the length of the alkyl tail of the liquid crystal part. Even the monolayer structure of the smectic phase $\left(\mathrm{S}_{\mathrm{A} 1}\right)$ is conserved by changing the tail length. This modification involves then more subtle rearrangements in the smectic phase.

4.1 ANALYSIS OF THE DIFFRACTION PATTERNS. - More information on the liquid crystal organization inside the smectic layer can be obtained from the analysis of the diffraction patterns. Figures 2 and 3 display the diffraction figures obtained at low temperature (the smectic order is saturated) for the mixtures protonated/deuterated on the backbone of PMA$\mathrm{OC}_{4} \mathrm{H}_{9}$ and $\mathrm{PMA}-\mathrm{OCH}_{3}$.

- PMA-OC ${ }_{4} \mathrm{H}_{9}$ presents a strong first order 001 and lower high orders (002 and 003) which are missing more or less following the type of labelling and the techniques (X-rays or neutron scattering).

- PMA-OCH 3 presents only one 001 Bragg peak independently of the techniques or the labelling type.

i) The analysis will be first built on the comparison of the 001 Bragg reflection of the two polymers.

It appears whatever the scattering techniques (X-rays or neutrons) and whatever the labelling (hydrogenated, deuterated on the tails of the mesogens or deuterated on the backbone), that the 001 Bragg peak of $\mathrm{PMA}-\mathrm{OC}_{4} \mathrm{H}_{9}$ is always largely stronger than that of PMA$\mathrm{OCH}_{3}$. The absolute intensity of the maximum of the $001 \mathrm{Bragg}$ reflection has been measured in units of the corresponding proton scattering deduced from the incoherent background. The 


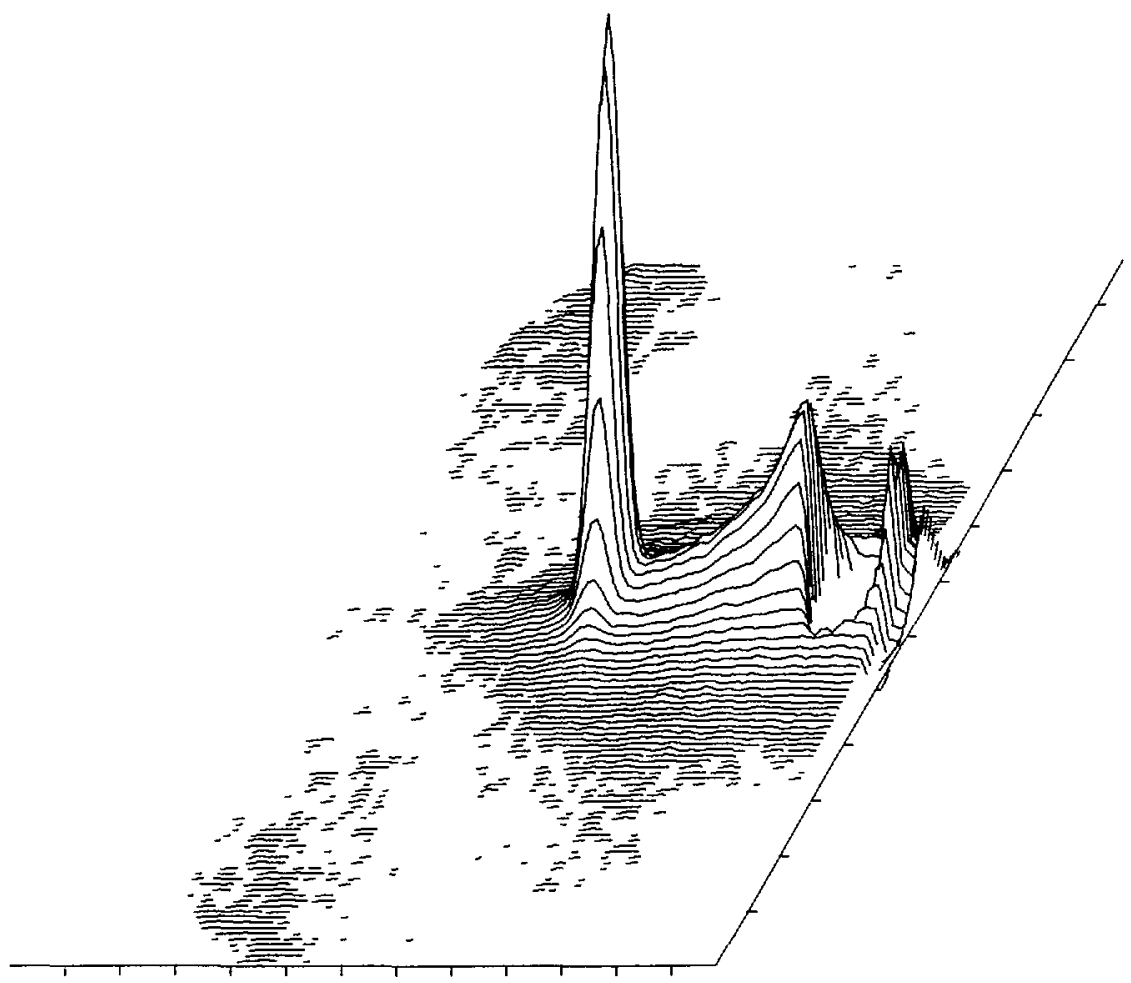

Fig. 2. - Diffraction pattern of the mixture $\mathrm{PMA}-\mathrm{OC}_{4} \mathrm{H}_{9} / \mathrm{PMA}(\mathrm{D})-\mathrm{OC}_{4} \mathrm{H}_{9}$ at low temperature. The magnetic field direction is horizontal.

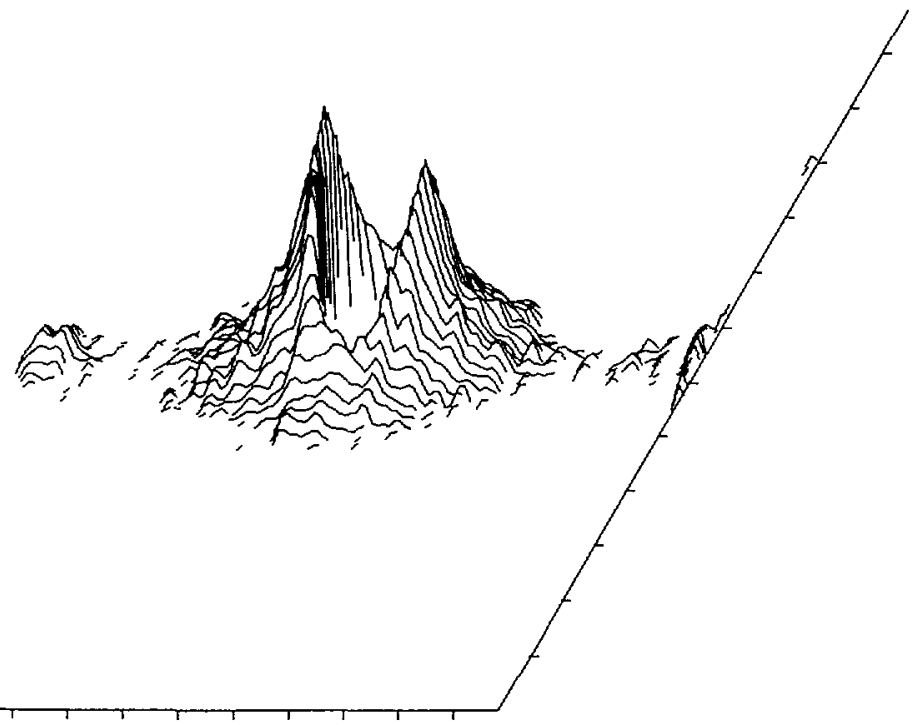

Fig. 3. - Diffraction pattern of the mixture $\mathrm{PMA}-\mathrm{OCH}_{3} / \mathrm{PMA}$ (D) $-\mathrm{OCH}_{3}$ obtained at low temperature. The magnetic field direction is horizontal. 
intensity of the maximum for the $\mathrm{PMA}-\mathrm{OC}_{4} \mathrm{H}_{9}$ peak is found to be 19 times greater than that of $\mathrm{PMA}-\mathrm{OCH}_{3}$.

If we consider the profile of the smectic layers as a periodic function of the density $\rho$ (of coherent scattering length for example), we can write

$$
\Delta \rho(z)=\sum F_{n} \cos 2 \pi n\left(\frac{z}{d}\right)
$$

where $\Delta \rho(z)$ is the density fluctuation at a point $z$ along the director and $d$ the smectic layer thickness. The integer $n$ determines the number of Bragg reflections we obtain in the reciprocal space, and the intensity of each Bragg reflection is given by $\left|F_{n}\right|^{2}$ since the Fourier transform of $\Delta \rho$ is :

$$
\mathrm{FT}(\Delta \rho(z)) \propto \sum_{n}\left|F_{n}\right|^{2} \delta\left(q_{z}-2 \pi \frac{n}{d}\right)
$$

Considering just one reflection (001) corresponds to approximate the smectic layers by a sinusoid where the amplitude $F_{001}$ characterizes the probability density to find the mesogen inside the layers [11].

So, just from the comparison of the 001 Bragg reflections of $\mathrm{PMA}-\mathrm{OCH}_{3}$ and neglecting the other low reflections of weak intensity of PMA-OC ${ }_{4} \mathrm{H}_{9}$, we can conclude that the layers of PMA$\mathrm{OCH}_{3}$ are formed with few localized mesogens that the polymer backbone can cross easily. This is the reason why the backbone anisotropy of $\mathrm{PMA}-\mathrm{OCH}_{3}$ is so weak. At the contrary,

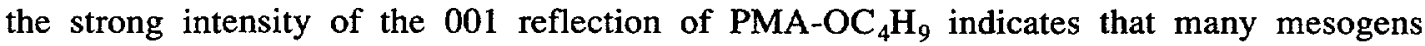
contribute to each layer. The walk of the polymer backbone is stopped by the strong density of mesogens localized in the layers and the exclusion of the polymer backbone from the mesogen zones increases the backbone anisotropy.

ii) The fact that $\mathrm{PMA}-\mathrm{OC}_{4} \mathrm{H}_{9}$, possesses in addition higher order of reflection $(002$ and 003) of weaker intensity indicates [12] that the density profile is more complicated that a

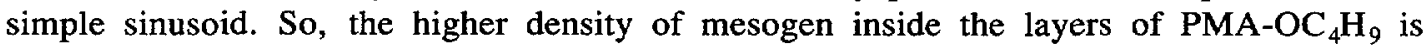
associated to a better structuration of the smectic layer.

4.2 EXPLANATION OF THE VARIATION OF THE MESOGEN DENSITY INSIDE THE LAYERS BY THE CHEMICAL STRUCTURE. - The formation of the smectic phase results of segregation between the different parts of the mesogens which is more or less strong following the importance of the aromatic and the alkyl parts. It is the reason why the long terminal group $\mathrm{OC}_{4} \mathrm{H}_{9}$ of $\mathrm{PMA}-\mathrm{OC}_{4} \mathrm{H}_{9}$ favorizes a well organized smectic phase whereas PMA-OCH ${ }_{3}$ offers a more blurred smectic arrangement.

\section{Conclusions.}

This article gives an example of the complementarity between the small angle neutron scattering data and the diffraction informations which are necessary to understand the double aspect (liquid crystal and polymer) of these compounds. Indeed, we have here demonstrated that the polymer backbone anisotropy is very sensitive to rather weak modifications of the smectic arrangement such as an increase of the length of the terminal alkyl group of the mesogens (this modification keeps the monolayer structure of the smectic phase). For this, the weak anisotropy of the backbone of $\mathrm{PMA}-\mathrm{OCH}_{3}$ is interpreted by the fact that the smectic layers of $\mathrm{PMA}^{-\mathrm{OCH}_{3}}$ are made with few aligned mesogens (weak 001 Bragg peak intensity) whereas the strong density of aligned mesogens in the layers of $\mathrm{PMA}-\mathrm{OC}_{4} \mathrm{H}_{9}$ defines a welldefined and dense smectic phase. In other words, the strength with which the smectic phase is 
established determines the confinement degree of the polymer backbone. This proposition is supported by another study [13] which shows the parallelism between the decrease of $R_{\mathbb{I}}$ and the increase of the 001 Bragg peak intensity as a function of temperature.

\section{References}

[1] GUINIER A., Théorie et Technique de la radiocristallographie (Dunod, Paris, 1964).

[2] GuinIER A., Fournet G., Small Angle Scattering of X-Rays (J. Wiley and Sons, New York, 1955).

[3] See for instance : Neutron, X-Ray and Light Scattering P. Lindner and T. Zemb Eds. (Elsevier Sc. Pub., 1991).

[4] Keller P., Carvalho B., Cotton J. P., Lambert M., Moussa M., Pepy G., J. Phys. Lett. France 46 (1985) L1065.

[5] KiRste R. G., OHM H. G., Makromol. Chem. Rapid Commun. 6 (1985) 179.

[6] Hardouin F., Noirez L., Keller P., Lambert M., Moussa F., Pepy G., Richard H., Mol. Cryst. Liq. Cryst. 155 (1988) 389.

[7] BouE F., Thesis, Univ. Paris XI (1982).

[8] NoIreZ L., Thesis, Univ. Paris XI (1989).

[9] DE Gennes P. G., Scaling Concepts in Polymer Physics (Cornell University Press, Ithaca and London, 1979) ;

Garvisch W., Brereton M. G., Fischer E. W., Polymer Bull. 4 (1981) 687 ;

Cotton J. P., Decker D., Benoit H., Farnoux B., Higgins J., Jannink G., Des Cloizeaux J., OBER R., PICOT C., Macromolecules 7 (1974) 863.

[10] Noirez L., Moussa F., Cotton J. P., Keller P., PePy G., J. Stat. Phys. 62 (1991) 997.

[11] De Gennes P. G., The Physics of Liquid Crystals (Clarendon Press, 1974) p. 290.

[12] Davidson P., Levelut A. M., Liq. Crystals 11 (1992) 469.

[13] Noirez L., Pegy G., Keller P., Benguigui L., J. Phys. II France 1 (1991) 821. 